\title{
Wideband Back-Cover Antenna Design Using Dual Characteristic Modes With High Isolation for $5 \mathrm{G}$ MIMO Smartphone
}

\author{
Wei Hu, Zhan Chen, Long Qian, Lehu Wen, Qi Luo, Senior Member, IEEE, Rui Xu, Wen Jiang \\ and Steven Gao, Fellow, IEEE
}

\begin{abstract}
A novel method of designing a wideband highisolated dual-antenna pair using dual characteristic modes (CMs) is presented for fifth-generation (5G) multiple-input multipleoutput (MIMO) smartphone applications. A set of orthogonal CMs resonating from the square-loop slot is first introduced and works for the lower band. Then, another set of orthogonal CMs resonating from the edge branches is introduced with a shared compact radiator and works for the higher band. In combination with two sets of degenerated CMs and a capacitive coupling feeding structure, the proposed dual-antenna pair achieves a broad impedance bandwidth and high isolation without the need for any external decoupling structures. Based on this dual-antenna pair, an $8 \times 8$ MIMO array is developed and integrated into the back cover of a smartphone, which realizes zero ground clearance on the system circuit board. To verify the design concept, prototypes of the antenna pair and MIMO array were fabricated and measured. It shows that experimental results agree well with the simulation results. More importantly, the presented $8 \times 8$ MIMO array has high isolation of more than $20 \mathrm{~dB}$ is achieved across the operating band of 3.3-3.8 GHz.
\end{abstract}

Index Terms-Back-cover antenna, characteristic mode (CM), dual-antenna pair, fifth-generation (5G), MIMO smartphone antenna.

\section{INTRODUCTION}

$\mathrm{M}$ ultiple-input multiple-output (MIMO) is a very effective technique for increasing the channel capacity, and it has been considered as one of the pivotal techniques for fifth-generation $(5 \mathrm{G})$ mobile communication systems [1]. The mutual coupling between MIMO antennas affects the

This work was supported in part by the National Natural Science Foundation of China under Grant No. 62071347, in part by EPSRC under Grants EP/N032497/1 and EP/S005625/1, and in part by the 111 Project of China. (Corresponding author: Wei Hu.)

W. Hu, Z. Chen, and W. Jiang are with the National Key Laboratory of Antennas and Microwave Technology, Xidian University, Xi'an, Shaanxi 710071, China. (e-mail: weihu.xidian@ieee.org).

L. Qian is with the School of Electronic Engineering and Computer Science, Queen Mary University of London, London E1 4NS, U.K. (e-mail: 1.qian@qmul.ac.uk).

L. Wen, and S. Gao are with the School of Engineering and Digital Arts, University of Kent, Canterbury CT2 7NT, U.K. (e-mail: s.gao@kent.ac.uk).

Q. Luo is with the School of Physics, Engineering and Computer Science, University of Hertfordshire, Hatfield AL10 9AB, U.K. (e-mail: qiluo@ieee.org).

R. Xu is with the School of Electrical and Electronic Engineering, Nanyang Technological University, Singapore 639798. (e-mail: rui.xu@ntu.edu.sg). orthogonality between different data streams and deteriorates the channel capacity in the communication systems [2], [3]. Therefore, how to ensure the high performance of MIMO antennas is a significant challenge, especially for the design of current 5G MIMO smartphone antennas.

In order to coexist with the original $2 \mathrm{G} / 3 \mathrm{G} / 4 \mathrm{G}$ antennas [4], [5], arranging $5 \mathrm{G}$ antennas on the long side or metal frame of the mobile phone is considered as a promising design scheme. Several decoupling methods have been proposed to reduce mutual coupling, such as space diversity [6], [7], neutralization line [8], [9], parasitic resonance decoupling [10], [11], self-isolation [12], polarization diversity [13], [14], and high-order mode decoupling [15]. Although high isolation is achieved in these MIMO antennas [6]-[15], a large space is occupied. To save the device space, terminal antennas using a shared radiator have attracted many research interests [16]-[24]. Some designs with multi-port antenna pairs have been proposed and studied utilizing the methods of polarization orthogonality [16]-[19], common mode and differential mode decoupling [20], [21], self-decoupling [22], and lumped components [23]. However, designing a MIMO antenna with simultaneous broadband, high isolation and compact size is still a challenge. Even in some special smartphone scenarios, zero ground clearance is required. To make full use of the limited space in the terminal device, MIMO antennas are integrated into the smartphone back cover [24]. However, due to the small distance between the radiator and the ground, the impedance bandwidth of the antenna is affected dramatically.

The theory of characteristic mode (TCM) is a promising antenna design theory [25], [26]. It provides another new view for antenna design. In [27], both antennas have wide impedance bandwidths of $130 \%$ based on TCM. In [28], a MIMO antenna for multi-mode $5 \mathrm{G}$ smartphone applications is designed based on TCM with dual broad bandwidth. A multi-band MIMO antenna for $5 \mathrm{G}$ and IoT devices is proposed, which is developed using the TCM to systematically design a MIMO antenna [29]. An ultra-wideband circularly polarized antenna using multiple characteristic modes $(\mathrm{CMs})$ is presented, the operating bandwidth is $92.3 \%\left(\left|\mathrm{~S}_{11}\right|<-10 \mathrm{~dB}, \mathrm{AR}<3 \mathrm{~dB}\right)$, and the $3-\mathrm{dB}$ gain bandwidth is $72.3 \%$ [30]. In addition, TCM also provides a set of basis currents to guarantee the orthogonality of the field patterns. Therefore, it has been utilized to realize various 
antennas for MIMO applications [31]-[36]. In [33], a miniaturized MIMO antenna using TCM is developed for smartphones with isolation better than $28 \mathrm{~dB}$. In [34], a pair of degenerated $\mathrm{CMs}$ is employed to achieve high port-to-port isolation. A wideband two-element MIMO antenna using metamaterial unit cells is presented with 25-dB isolation [35]. In [36], characteristic electric field distributions from CMA are used to find the feed position in a tri-modal patch antenna. However, few of them have the characteristics of broadband, high isolation, low profile, and low ground clearance simultaneously. In the design of a multiport MIMO antenna, individual excitation of each $\mathrm{CM}$ becomes difficult as more CMs will be involved and intertwined with each other.

In this paper, a wideband and high-isolated back-cover antenna pair for smartphones is proposed. To the authors' knowledge, to date there is no reported design using two sets of $\mathrm{CMs}$ to design the back-cover MIMO antenna pairs with broadband, high isolation, low profile, and zero ground clearance simultaneously. The first set of CMs is designed and generated in the inner slot of the ring at $3.4 \mathrm{GHz}$, and the second set of $\mathrm{CMs}$ is designed and generated on the edge branch at $3.8 \mathrm{GHz}$. By combining two sets of CMs and introducing a pair of feeding branches, a wide impedance bandwidth covering 3.3-3.8 GHz and high isolation of $20 \mathrm{~dB}$ can be obtained without using any decoupling structures. In addition, a low profile with the thickness of only $1.6 \mathrm{~mm}$ is achieved. To validate the design concept, four dual-antenna pairs are placed at the corners of the smartphone back cover with high isolation. Then, these antenna pairs are integrated into the back cover of the smartphone to achieve zero ground clearance on the system circuit board, which reserves a complete system circuit board for other components in the smartphone. The proposed antenna pair and antenna array were also fabricated and measured. Experimental results agree well with the simulations. It shows that high isolation of more than $20 \mathrm{~dB}$ is achieved across the operating band of 3.3-3.8 GHz for the fabricated $8 \times 8$ MIMO array.

\section{Patch Design of Characteristic Mode}

In this section, CMA is first used to research on a low-profile patch antenna to form two sets of orthogonal CMs. Then, these two sets of CMs are elaborately combined, which builds an important foundation to the proposed wideband high-isolation MIMO antenna.

\section{A. The theory of characteristic mode}

According to TCM, the characteristic mode fields form a weighted orthogonal set over a conductor surface and the sphere at infinity [27] [33]. The total surface field distribution of an antenna can be obtained by the superposition of each mode field. In TCM, $\alpha_{n}$ is a weighting factor defined as modal weighting coefficient (MWC). The MWC reflects the mode contribution of the total field density, and its expression is:

$$
\alpha_{n}=\frac{V_{n}}{1+j \lambda_{n}}=\frac{\left\langle\mathbf{J}_{n}, \mathbf{E}^{i}\right\rangle}{1+j \lambda_{n}}
$$

where $V_{n}$ reflects the coupling of external excitation and

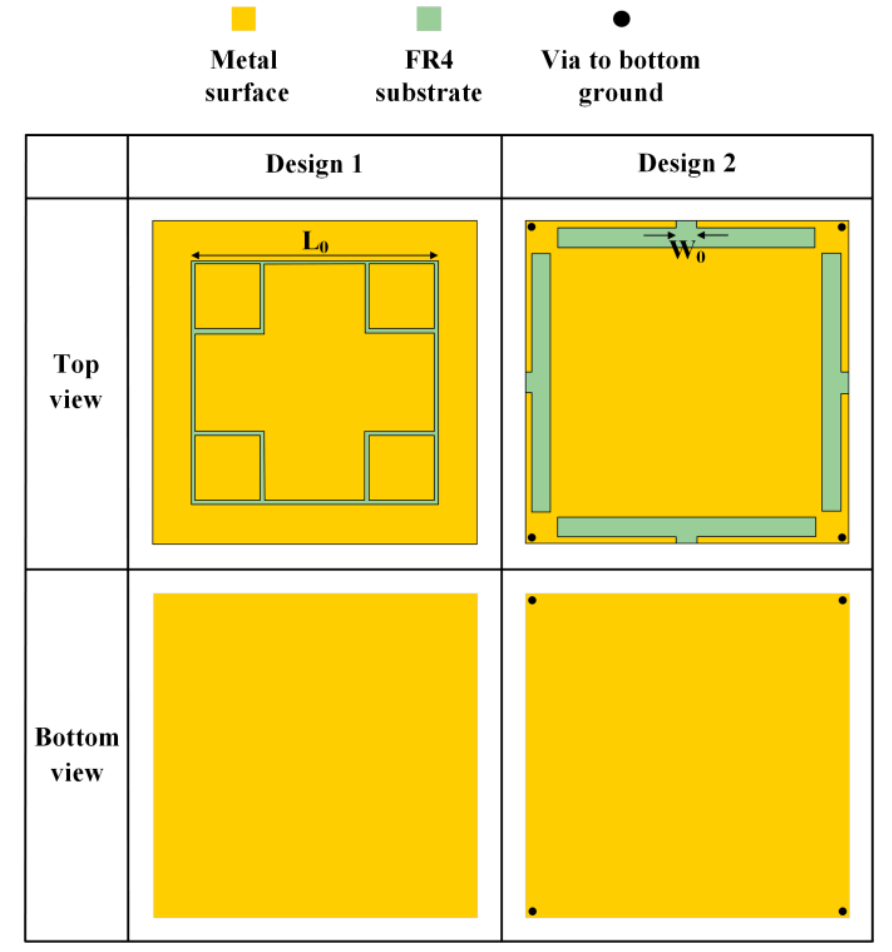

Fig. 1. Geometry of preliminary designs in $\mathrm{CMA}\left(\mathrm{L}_{0}=20.6\right.$ and $\mathrm{W}_{0}=1.9$, all dimensions in $\mathrm{mm}$ ).

characteristic current, which is defined as the mode excitation coefficient (MEC).

Each CM corresponds to a characteristic value $\lambda_{n}$, which represents the ratio of reactive power to radiated power. Modal significance (MS) is closely related to $\lambda_{n}$, which is denoted as:

$$
\mathrm{MS}_{n}=\frac{1}{\left|1+j \lambda_{n}\right|}
$$

$\mathrm{MS}_{n}$ is independent of the excitation source, and only related to the shape of the conductor. It indicates the potential ability of a $\mathrm{CM}$ working in certain bands. TCM defines a series of characteristic currents on the antenna. Each characteristic current has a corresponding characteristic E-field. The phase difference between the characteristic E-field and characteristic current is defined as the characteristic angle (CA) $\beta_{n}$. When $\beta_{n}=180^{\circ}$, the relevant $\mathrm{CMs}$ will be in resonance state.

$$
\beta_{n}=180^{\circ}-\arctan \lambda_{n}
$$

\section{B. Design of Initial Two CMs Sets}

In this section, two sets of new CMs are proposed. Fig. 1 shows the geometries of the two original design models (Design 1 and Design 2) for characteristic mode analysis (CMA). Design 1 and Design 2 occupy different areas of the same radiating patch. The metal structure is printed on the top of an FR4 substrate with the size of $27 \mathrm{~mm} \times 27 \mathrm{~mm} \times 1.6 \mathrm{~mm}$, and another complete metal ground is printed on the bottom of the substrate. In Design 1, the upper metal surface and the lower metal surface are not connected to each other, $\mathrm{L}_{0}$ is the side length of the inner square-loop slot. In Design 2, four metalized via holes connect the top layer to the bottom layer of the substrate at the corners, $\mathrm{W}_{0}$ is the slot length between the edge 


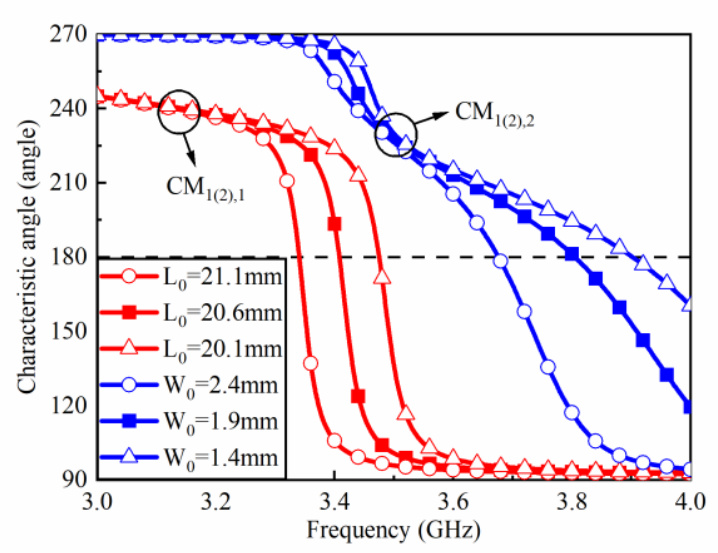

Fig. 2. Effects of $\mathrm{L}_{0}$ and $\mathrm{W}_{0}$ on the first two CMs' CAs of Design 1 and Design 2.

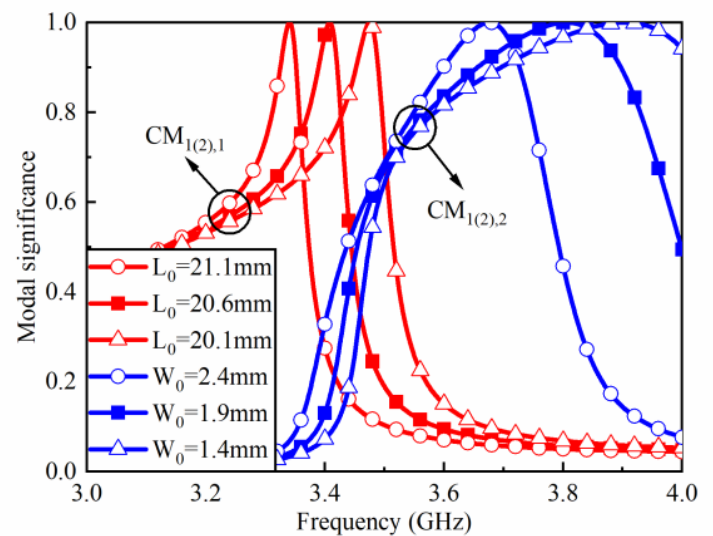

Fig. 3. Effects of $\mathrm{L}_{0}$ and $\mathrm{W}_{0}$ on the first two CMs' MSs of Design 1 and Design 2.

branches.

CA and MS are two important parameters for CMA, which are obtained by the commercial software FEKO. For Design 1 and Design 2, the simulated CAs and MSs of two most relevant CMs in CMA are presented in Fig. 2 and Fig. 3. In these figures, $\mathrm{CM}_{m, n}$ is the $m^{\text {th }}$ characteristic mode of Design $n$. It can be observed that in Design 1, two CMs resonate around 3.4 GHz. While in Design 2, two CMs resonate near 3.8 GHz. Due to the symmetry of the structure, two CMs of Design 1 or Design 2 are equal. Therefore, the following relations can be obtained:

$$
\begin{aligned}
\mathrm{CA}_{1,1(2)} & =\mathrm{CA}_{2,1(2)} \\
\mathrm{MS}_{1,1(2)} & =\mathrm{MS}_{2,1(2)}
\end{aligned}
$$

where $\mathrm{CA}_{m, n}$ and $\mathrm{MS}_{m, n}$ represents the characteristic angle and modal significance of $\mathrm{CM}_{m, n}$, respectively.

According to TCM, different CMs are orthogonal to each other. To investigate the operation mechanism of Designs 1 and 2, the characteristic E-fields at $3.4 \mathrm{GHz}$ and $3.8 \mathrm{GHz}$ are presented in Fig. 4. As shown in Fig. 4(a), strong characteristic E-field distribution of $\mathrm{CM}_{1,1}$ is observed in the center of the square-ring slot along the $x$-axis (Region $\mathrm{A}_{1}$ ) at $3.4 \mathrm{GHz}$. Fig. 4(b) shows that the E-field of $\mathrm{CM}_{2,1}$ is concentrated around the center of square-ring slot along the $y$-axis (Region $\mathrm{B}_{1}$ ). These two figures denote that the orthogonal set of resonances at 3.4 $\mathrm{GHz}$ comes from the square-ring slot. At the frequency of 3.8 (a)

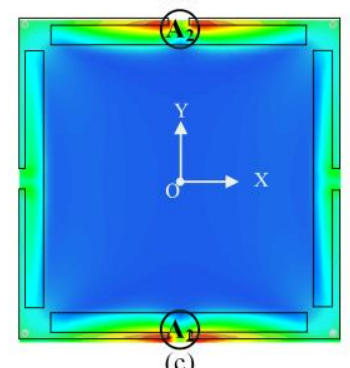

(c)

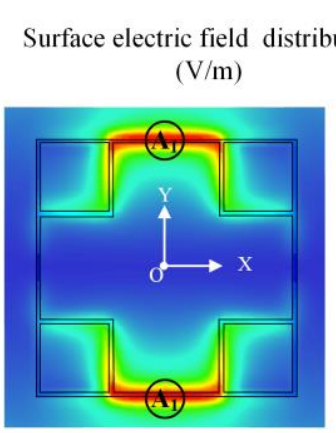

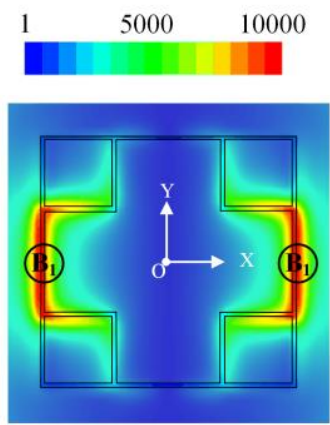

(b)

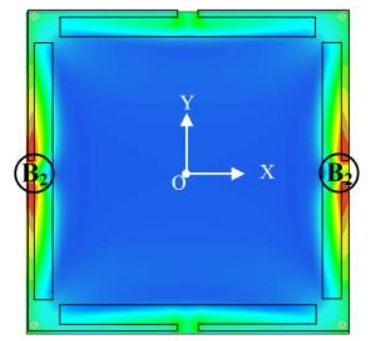

(d)
Fig. 4. Simulated characteristic E-field distribution of proposed antenna designs. (a) $\mathrm{CM}_{1,1}$ at $3.4 \mathrm{GHz}$. (b) $\mathrm{CM}_{2,1}$ at $3.4 \mathrm{GHz}$. (c) $\mathrm{CM}_{1,2}$ at $3.8 \mathrm{GHz}$. (d) $\mathrm{CM}_{2,2}$ at $3.8 \mathrm{GHz}$.

$\mathrm{GHz}$, the E-field of $\mathrm{CM}_{1,2}$ distributes on the edge branches along the $x$-axis (Region $\mathrm{A}_{2}$ ) as shown in Fig. 4(c). In Fig. 4(d), E-field maxima of $\mathrm{CM}_{2,2}$ are observed around the edge branches along the $y$-axis (Region $\mathrm{B}_{2}$ ). These two figures denote that the orthogonal set of resonances at $3.8 \mathrm{GHz}$ originates from the edge branch.

Based on these characteristic E-field distributions, the influences of the related geometrical dimensions on the CAs and MSs are further investigated. Simulations have been performed to evaluate the variations of CAs and MSs with different $\mathrm{L}_{0}$ and $\mathrm{W}_{0}$, and the results are given in Fig. 2 and 3. In these figures, $\mathrm{L}_{0}$ is the side length of the inner slot of the square loop in Design 1, and $\mathrm{W}_{0}$ is the slot length between the edge branches in Design 2. It can be seen when $\mathrm{L}_{0}$ increases from 20.1 to $21.1 \mathrm{~mm}$, the frequencies of $\mathrm{CM}_{1(2), 1}$ decrease to 3.3 $\mathrm{GHz}$. When $\mathrm{W}_{0}$ increases, $\mathrm{CM}_{1(2), 2}$ shifts to lower frequencies. Therefore, the resonances of Design 1 and Design 2 can be effectively controlled by adjusting $\mathrm{L}_{0}$ and $\mathrm{W}_{0}$. As can be seen, the simulated results are also consistent with the observed characteristic E-field distributions in Design 1 and Design 2.

\section{Combination CM Design Sets}

The structure of Design 1 produces radiation through the internal slot of the patch, and the strong magnitude of the characteristic E-field (Region $\mathrm{A}_{1}$ and $\mathrm{B}_{1}$ ) at $3.4 \mathrm{GHz}$ can be found at the midpoint of the adjacent side. The structure of Design 2 produces radiation through the edge branches of the patch, and the strong magnitude of the characteristic E-field (Region $\mathrm{A}_{2}$ and $\mathrm{B}_{2}$ ) at $3.8 \mathrm{GHz}$ is located at the midpoint of the adjacent side. In this section, two structures are integrated into one antenna unit for space saving and efficient excitation.

The evolution process of Design 3 is shown in Fig. 5, where Design 1 and Design 2 are elaborately combined into Design 3. 


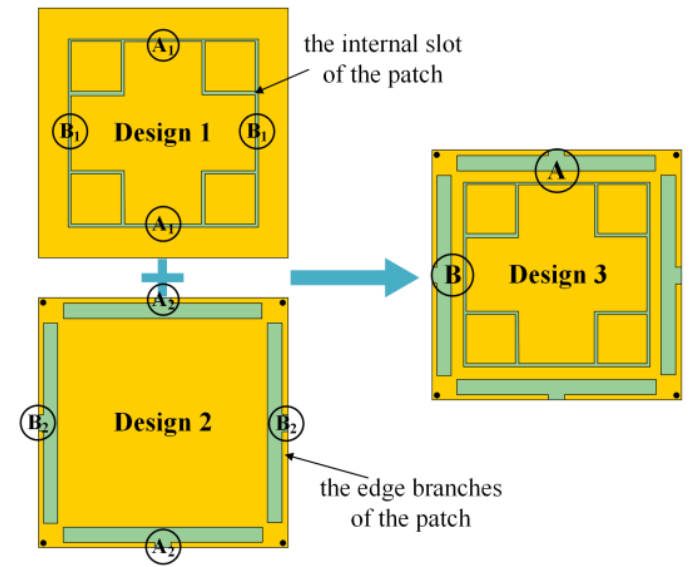

Fig. 5. The evolution process of combined Design 3.

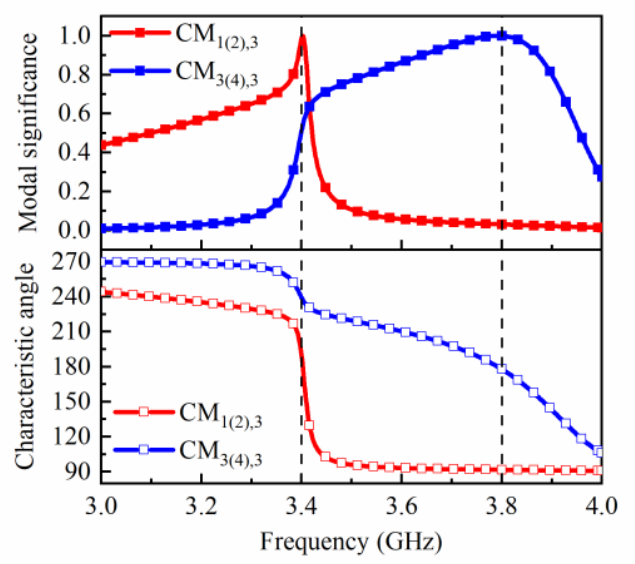

Fig. 6. Simulated MSs and CAs on the first four CMs of proposed Design 3.

The simulated CAs and MSs of the four most relevant CMs of the proposed Design 3 are presented in Fig. 6. As shown in the figure, the simulated results of Design 3 agree well with the CAs and MSs of Design 1 and Design 2 shown in Fig. 2 and 3. Therefore, $\mathrm{CM}_{1(2), 3}$ corresponds to $\mathrm{CM}_{1(2), 1}$, and $\mathrm{CM}_{3(4), 3}$ is approximately equal to $\mathrm{CM}_{1(2), 2}$, which verifies the effectiveness of our combination design. It is very promising to obtain a broadband and high-isolated MIMO dual-antenna pair by exciting the four orthogonal CMs in Design 3.

\section{Low PROFILE $2 \times 2$ MIMO ANTENNA PROTOTYPE}

Based on the above analysis of the dual-set orthogonal CMs and the detailed feeder optimization, a low-profile wideband high-isolated dual-antenna pair is proposed in this section for 5G MIMO applications in N78 band. Then, the proposed antenna pairs are experimentally verified, and the measured results are in good agreement with the simulation.

\section{A. Proposed Dual-Antenna Pair Structure}

The detailed configuration of the proposed antenna pair is shown in Fig. 7. The antenna pair is printed on the top of an FR4 substrate (relative permittivity 4.4 , loss tangent 0.02 , and thickness $1.6 \mathrm{~mm}$ ) with a compact size of $27 \mathrm{~mm} \times 27 \mathrm{~mm}$. Note that the bottom ground has the same size as the top one. The simulated S-parameters of the proposed antenna pair are
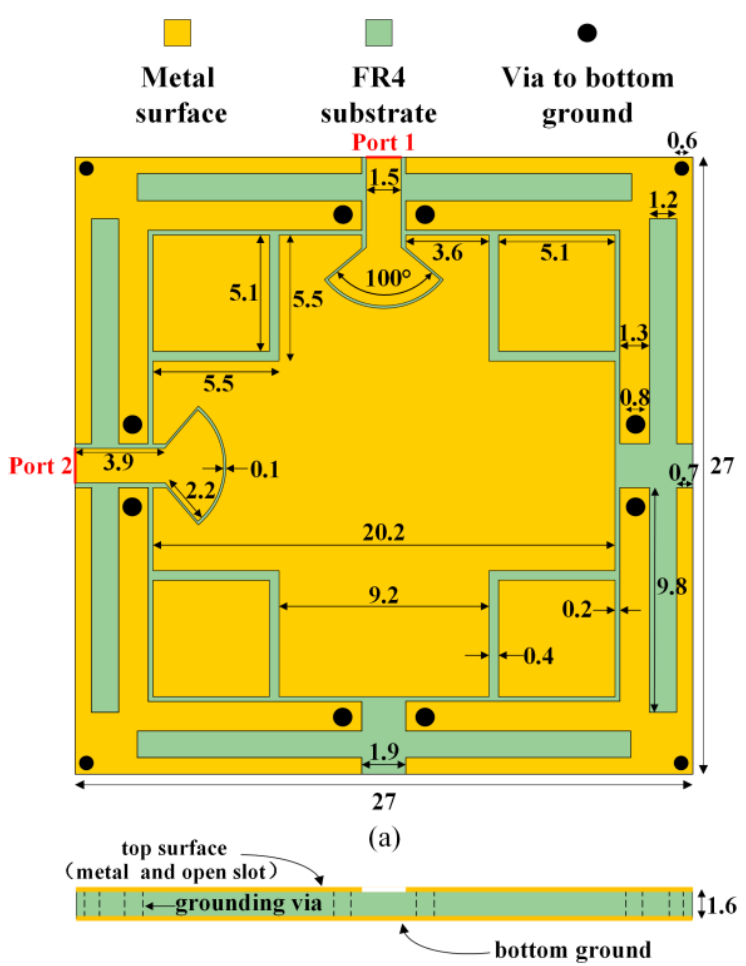

(b)

Fig. 7. Geometry of the low-profile high-isolated MIMO antenna pair (all dimensions in mm). (a) Top view. (b) Cross-sectional view.

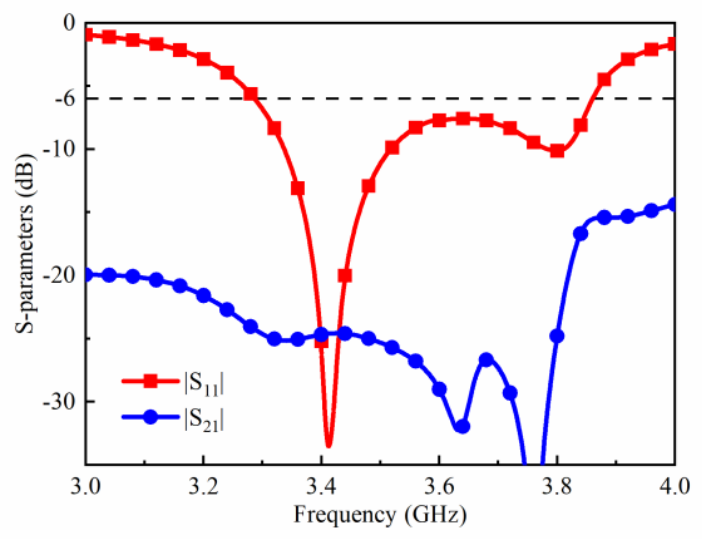

Fig. 8. Simulated S-parameters of the proposed antenna pair.

shown in Fig. 8. The simulated results are obtained by using the high-frequency structure simulator (HFSS). Due to the symmetry of the antenna pair, only $\left|S_{11}\right|$ and $\left|S_{21}\right|$ are given for brevity. It can be seen that the dual-antenna pair can cover 3.3-3.8 GHz. Meanwhile, because the two CMs excited by port 1 are orthogonal with the two CMs excited by port 2, high isolation of more than $24 \mathrm{~dB}$ can be achieved within the operation bandwidth. The expected CMs are well excited, which can ensure the required bandwidth and high isolation.

The E-field distributions of the dual-antenna pair are shown in Fig. 9, it can be clearly seen that the orthogonality of the excited working modes and the surface E-field distribution agree well with the previous dual sets of CMs. When port 1 is activated, the working E-field distribution of the antenna pair at $3.4 \mathrm{GHz}$ and $3.8 \mathrm{GHz}$ are almost the same as the characteristic 


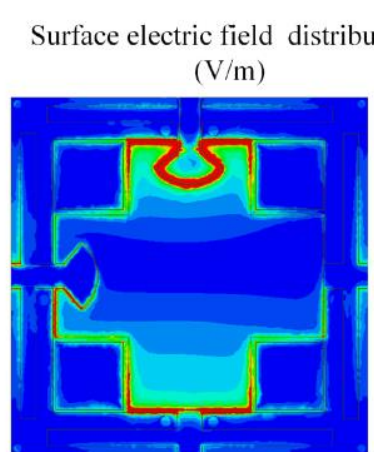

(a)

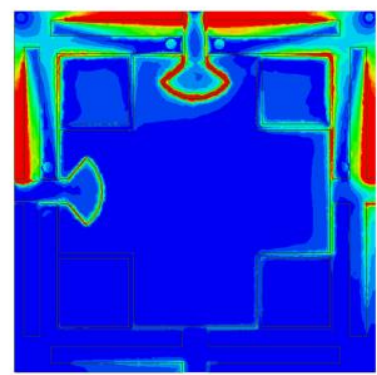

(c)

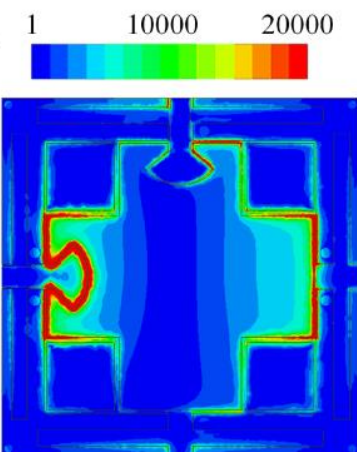

(b)

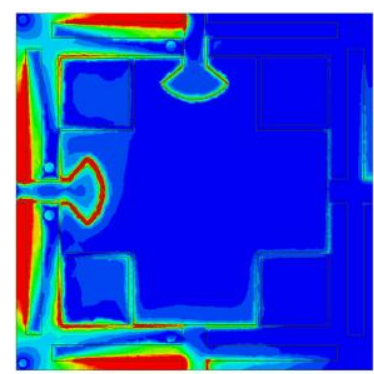

(d)
Fig. 9. Simulated E-field distribution of the proposed antenna pair. (a) Excitation by port 1 at $3.4 \mathrm{GHz}$. (b) Excitation by port 2 at $3.4 \mathrm{GHz}$. (c) Excitation by port 1 at $3.8 \mathrm{GHz}$. (d) Excitation by port 2 at $3.8 \mathrm{GHz}$.

E-field distribution of $\mathrm{CM}_{1,1}$ and $\mathrm{CM}_{1,2}$. Similarly, the working E-field distribution by port 2 of the antenna pair at $3.4 \mathrm{GHz}$ and $3.8 \mathrm{GHz}$ are also similar to the characteristic E-field distribution of $\mathrm{CM}_{2,1}$ and $\mathrm{CM}_{2,2}$. The design goals of wide impedance bandwidth and high isolation can be expected based on two sets of CMs in the presented antenna pair.

\section{B. Evolution of Feed Mechanism}

In TCM, how to excite the antenna to get the expected CMs is very critical. There are two design options for exciting CMs: one employs inductive coupling elements (ICE), and the other uses capacitive coupling elements (CCE) [37]. Since the strong characteristic E-fields are located at the midpoint of the adjacent sides (Regions A and B) in Design 3, whereas ICE is difficult to stimulate the radiation mode of slot, so two CCEs are chosen placed at the E-field intensity maximum: CCE1 and CCE2. The incident E-fields produced by CCE are denoted as $\mathbf{E}_{\mathrm{CCE} 1}$ and $\mathbf{E}_{\mathrm{CCE} 2}$, which can be obtained by

$$
\begin{aligned}
& \mathbf{E}_{\mathrm{CCE} 1} \approx \frac{\left\langle\mathbf{J}_{1}, \mathbf{E}_{\mathrm{CCE} 1}^{i}\right\rangle}{1+j \lambda_{1}} \mathbf{E}_{1} \\
& \mathbf{E}_{\mathrm{CCE} 2} \approx \frac{\left\langle\mathbf{J}_{2}, \mathbf{E}_{\mathrm{CCE} 2}^{i}\right\rangle}{1+j \lambda_{2}} \mathbf{E}_{2}
\end{aligned}
$$

where the $\mathbf{E}_{1}$ and $\mathbf{E}_{2}$ refer to the characteristic E-field. Since each set of CMs proposed in this paper is orthogonal, $\mathbf{E}_{1}$ is perpendicular to $\mathbf{E}_{2}$. The overall working modes of the antenna are also orthogonal to each other:

$$
\langle\mathbf{E C C E} 1, \mathbf{E C C E} 2\rangle=0
$$

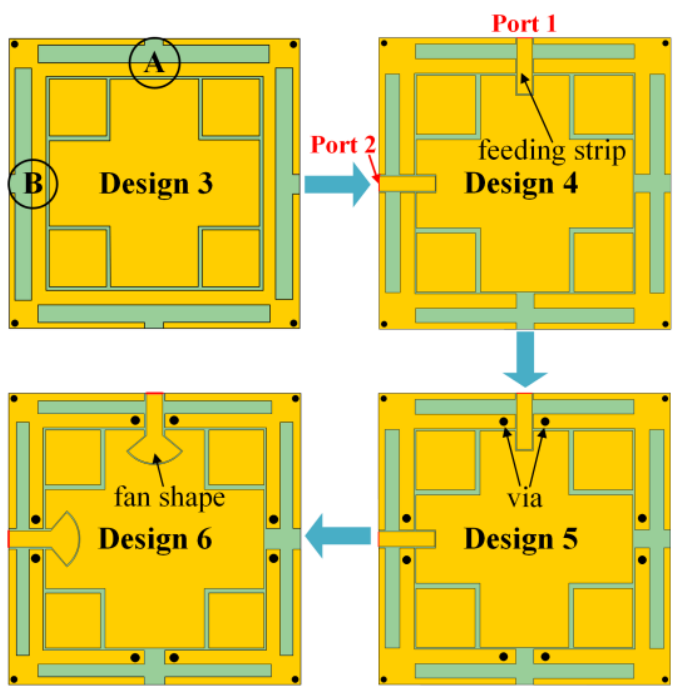

Fig. 10. The final antenna pair evolution process.

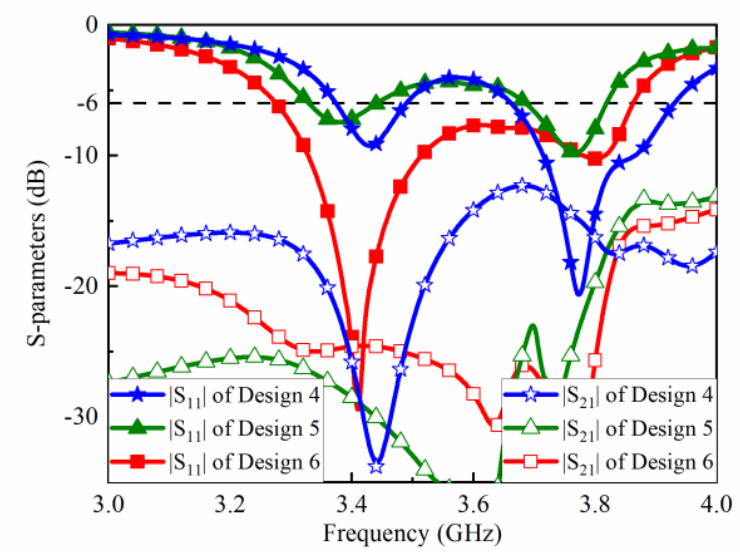

Fig. 11. Comparison of reflection coefficient of the dual-antenna pair proposed in design 4-design 6 .

Therefore, a set of CMs can achieve high isolation of MIMO antenna. The use of two sets of CMs can achieve high isolation within a wide bandwidth.

The final antenna pair evolution process is shown in Fig. 10. Fig. 11 shows the reflection coefficients of Design 4 to Design 6. By arranging two ports with an orthogonal configuration, $\mathrm{CM}_{1(3), 3}$ and $\mathrm{CM}_{2(4), 3}$ can be excited respectively, and a broadband antenna pair with high isolation can be realized. Based on the above design concept, Design 4 is proposed with an open-circuited feeding strip in the center of the adjacent side (Regions A and B in Design 3), which successfully excites the modes proposed in CMA. However, additional modes are observed in Design 4 with low isolation.

To avoid the additional modes, a pair of via holes is employed to connect the branch to the ground in Design 5. To reduce the influences of asymmetry, the ground via holes on each side are introduced. Finally, a fan-shaped structure at the end of the feeding strip is introduced to improve the impedance matching compared to Design 5 [38] [39]. The inductance introduced by the fan-shaped structure offsets the capacitance introduced by the CCE. The final antenna pair in Design 6 can cover 3.3-3.8 GHz for $\left|\mathrm{S}_{11}\right|<-6 \mathrm{~dB}$. In addition, after 


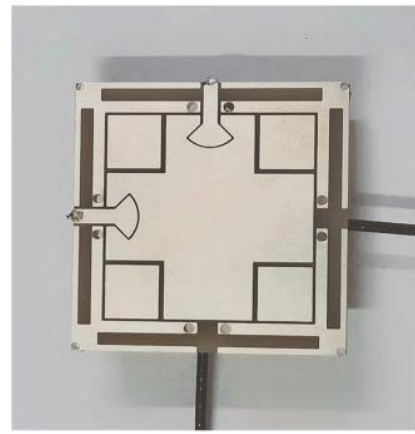

(a)

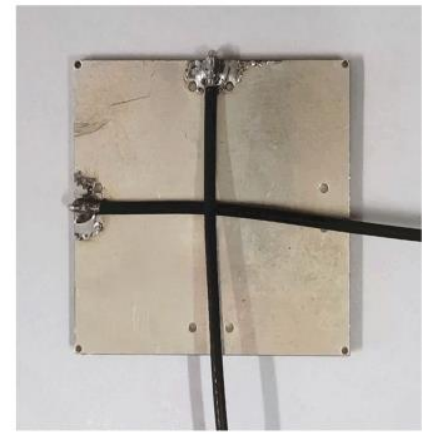

(b)
Fig. 12. Photographs of the fabricated antenna pair. (a) Top view. (b) Bottom view.

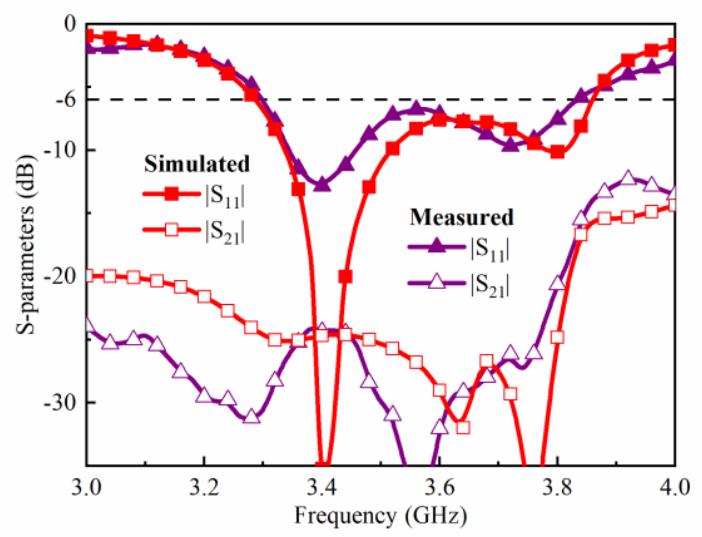

Fig. 13. Simulated and measured S-parameters of the fabricated antenna pair.

suppressing the additional modes, the isolation is nearly not affected because of the orthogonality of two sets of CMs.

\section{Experimental Verification of Antenna Pair}

To verify the antenna design based on CMA, the dual-antenna pair were fabricated and tested. The photographs of the fabricated antenna pair are shown in Fig. 12. The measured S-parameters of the fabricated prototype are shown in Fig. 13. It can be seen that the measured results agree well with the simulated results. This may be caused by the unstable permittivity of the low-cost FR4 substrate. The resonance at the higher frequency shifts to the lower frequency, and the $\left|S_{21}\right|$ curve slightly moves to the lower frequency. Despite that, the antenna pair can still cover 3.3-3.8 GHz with the $\left|\mathrm{S}_{11}\right|$ lower than $-6 \mathrm{~dB}$. The isolation between the two ports is higher than $20 \mathrm{~dB}$.

The envelope correlation coefficient (ECC) is an important parameter to evaluate the diversity performance between two MIMO antenna far-field patterns [40]. Fig. 14 shows that the simulated and calculated ECCs of the antenna pair, which are less than 0.01 in the operating bandwidth. Due to the orthogonality, extremely low ECC is achieved even though two ports are closely placed. The simulated and measured radiation efficiencies of the antenna pair are also shown in Fig. 14. The antenna efficiencies excited by port 1 and port 2 are both better than $45 \%$. The obtained results are promising for practical terminal applications.

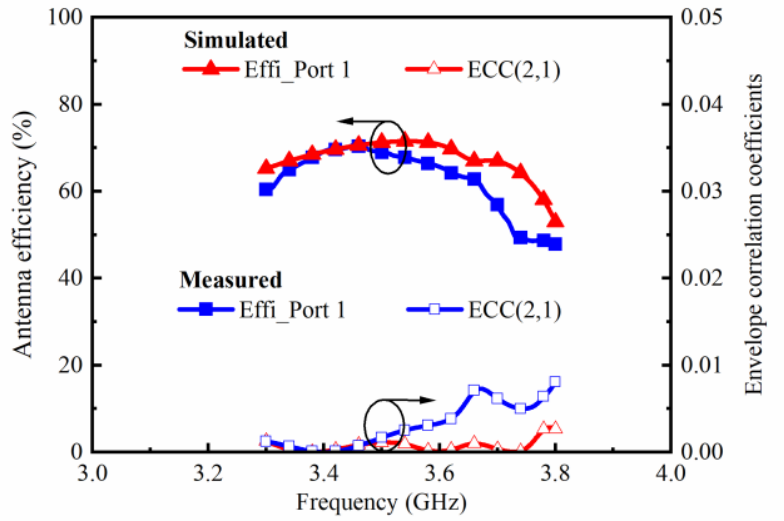

Fig. 14. Simulated and measured antenna efficiencies and ECCs of the fabricated Antenna pair.

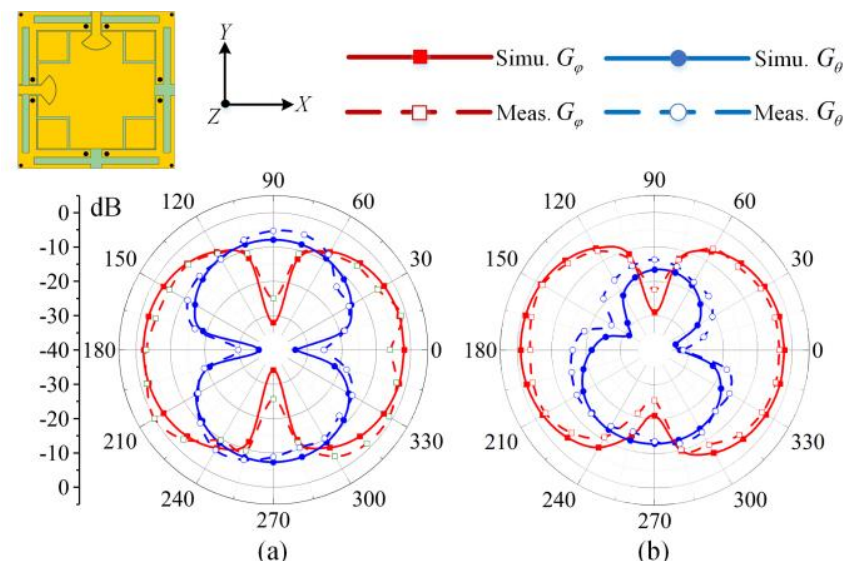

(a)

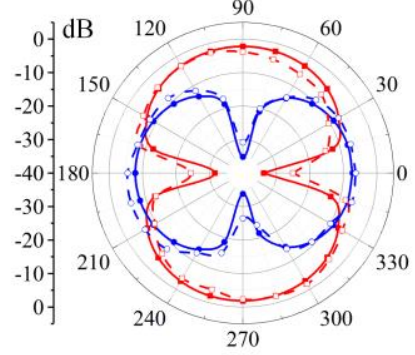

(c)

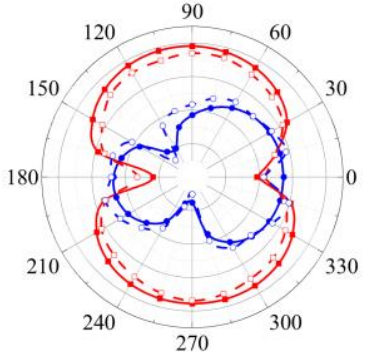

(d)
Fig. 15. Simulated and measured radiation patterns in the $x y$-plane of the fabricated antenna pair. (a) Port 1 at $3.4 \mathrm{GHz}$. (b) Port 1 at $3.8 \mathrm{GHz}$. (c) Port 2 at $3.4 \mathrm{GHz}$. (d) Port 2 at $3.8 \mathrm{GHz}$.

Fig. 15 shows the simulated and measured radiation patterns at 3.4 and $3.8 \mathrm{GHz}$, respectively. The measured radiation patterns agree well with the simulated results, and the radiation pattern is quasi-omnidirectional, which is very suitable for terminal device applications. Due to the symmetry of the structure, it can be seen that the patterns of port 1 and port 2 show a good orthogonality to each other when working at the same frequency.

\section{IV. $8 \times 8$ MIMO BACK COVER ANTENNA DESIGN AND IMPLEMENTATION}

In this section, four dual-antenna pairs are integrated at four corners of the smartphone back cover to obtain high isolation. Then, user hand grip effects are researched. Finally, the 


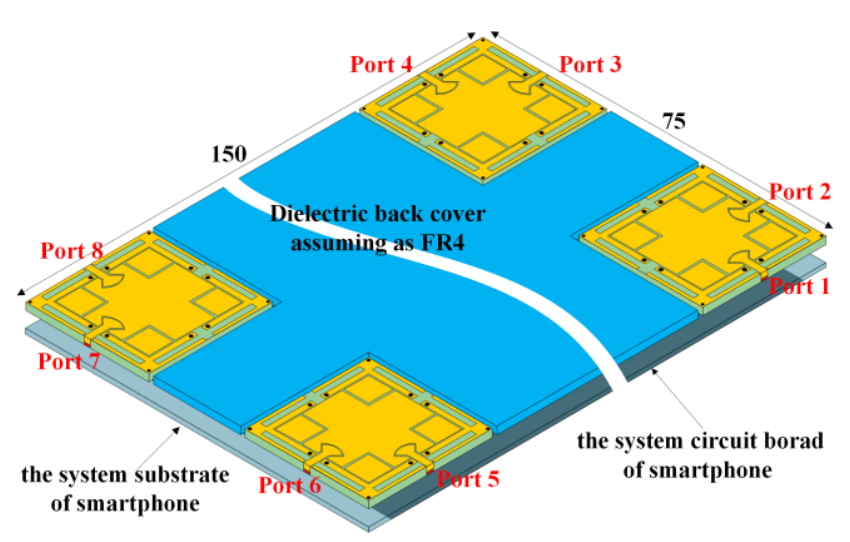

(a)

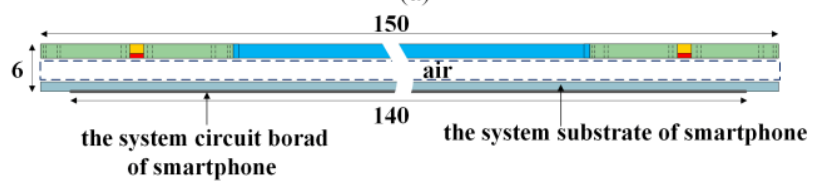

(b)

Fig. 16. Geometry of four antenna pairs backed by a dielectric cover with the system circuit board of 5G smartphone. (a) 3D view. (b) Cross-sectional view.

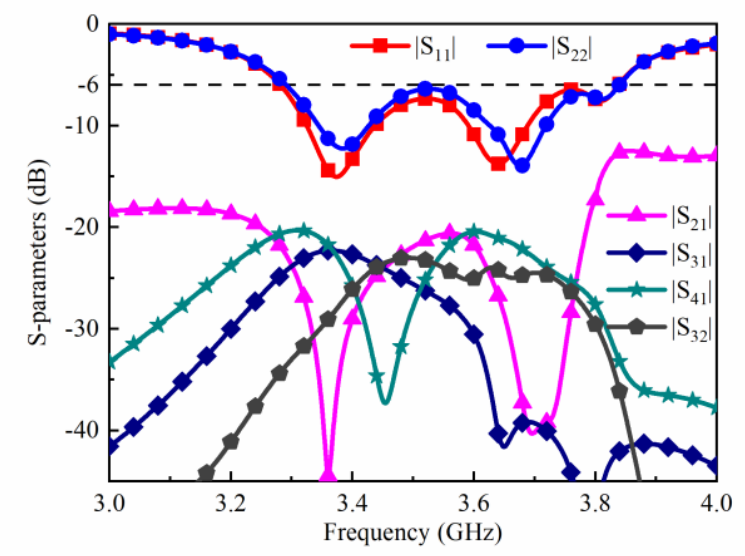

Fig. 17. Simulated S-parameters for $8 \times 8$ MIMO antennas backed by a dielectric cover with the system circuit board.

performance of the proposed antenna is compared with the current state of the art.

\section{A. $8 \times 8$ MIMO Back Cover Antenna Design}

The 3D view and cross-sectional view of the integration design are shown in Fig. 16. Another FR4 is inserted as the back cover for the smartphone with a size of $150 \mathrm{~mm} \times 75$ $\mathrm{mm} \times 1.6 \mathrm{~mm}$. Considering the machining error, four corners of $28 \mathrm{~mm} \times 28 \mathrm{~mm}$ are removed out of the dielectric cover back. Four antenna pairs are arranged at the four corners of the dielectric back cover. In order to simulate the real environment inside the smartphone, the system circuit board and a layer of 1 mm-thick FR4 substrate are included in the simulation. In addition, $5 \mathrm{~mm}$ clearance for $2 \mathrm{G} / 3 \mathrm{G} / 4 \mathrm{G}$ antennas at both ends of the system circuit board is reserved. Finally, the back-cover antenna is placed on the top of the system circuit board, and the entire thickness of $6 \mathrm{~mm}$.

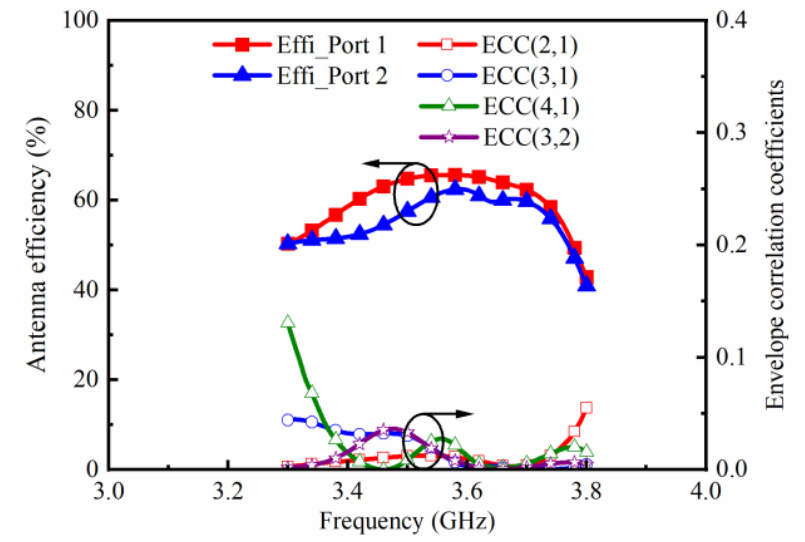

Fig. 18. Simulated antenna efficiencies and ECCs for $8 \times 8$ MIMO antennas backed by a dielectric cover with the system circuit board.

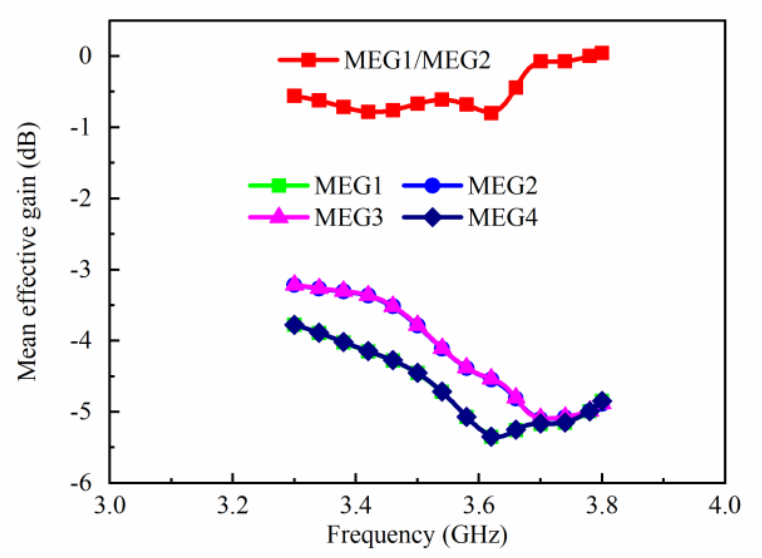

Fig. 19. Simulated MEGs of the proposed $8 \times 8$ MIMO antennas system backed by a dielectric cover with the system circuit board.

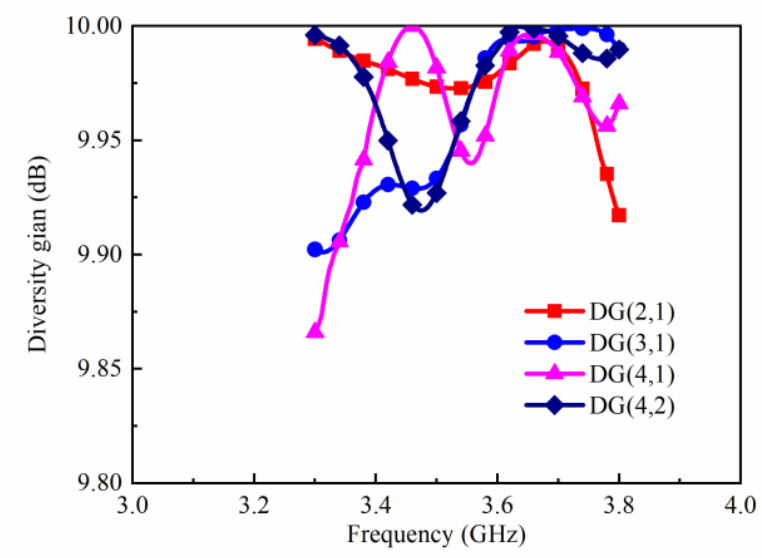

Fig. 20. Simulated DGs of the proposed $8 \times 8$ MIMO antennas system backed by a dielectric cover with the system circuit board.

The simulated S-parameters for the $8 \times 8$ MIMO antennas backed by a dielectric cover with the system circuit board are shown in Fig. 17. Due to the symmetry of the antenna structure, only $\left|S_{11}\right|$ and $\left|S_{22}\right|$ of reflection coefficient are given for clarity. As can be seen, the $8 \times 8$ MIMO antennas can cover the operating band of 3.3-3.8 GHz. The simulated transmission coefficients are higher than $18 \mathrm{~dB}$. The simulated antenna efficiency and ECCs for the $8 \times 8$ MIMO antenna backed by a 


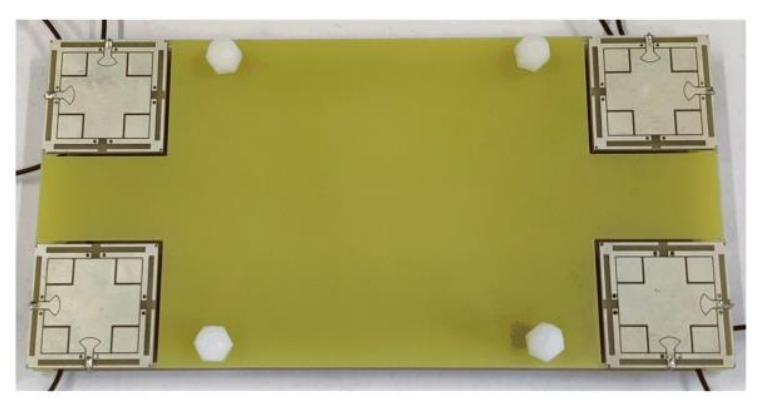

Fig. 21. Photographs of the geometry of $8 \times 8$ MIMO antennas backed by a dielectric cover with the system circuit board of the $5 \mathrm{G}$ smartphone.

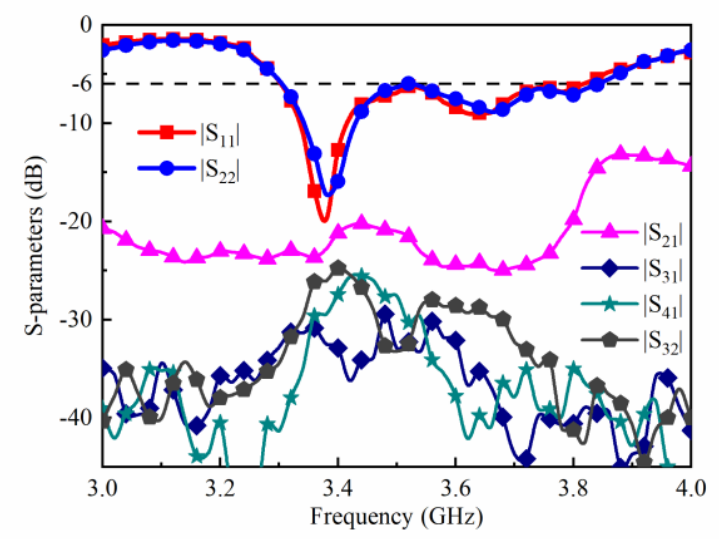

Fig. 22. Measured S-parameters of fabricated $8 \times 8$ MIMO antennas.

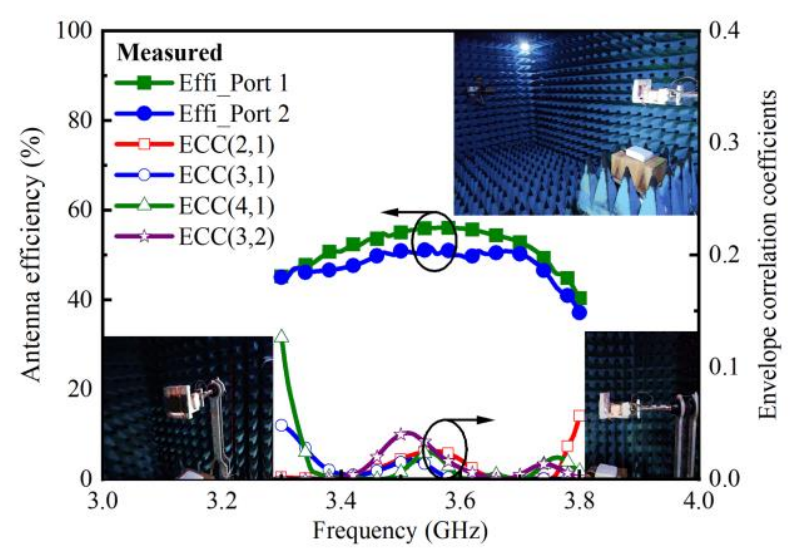

Fig. 23. Measured antenna efficiencies and ECCs for the fabricated $8 \times 8$ MIMO antennas.

dielectric cover with the system circuit board are shown in Fig. 18. The antenna efficiency is greater than $40 \%$, and the ECCs of the antenna array are lower than 0.13 .

The mean effective gain (MEG) ratio is another important parameter to evaluate the diversity performance, which is defined as the ratio of the mean receive power to the mean incident power [41]. The ratios of the mean effective gain should satisfy the following criteria in order to guarantee a good channel characteristic:

$$
\left|\frac{\mathrm{MEG}_{i}}{\mathrm{MEG}_{j}}\right| \approx 1
$$

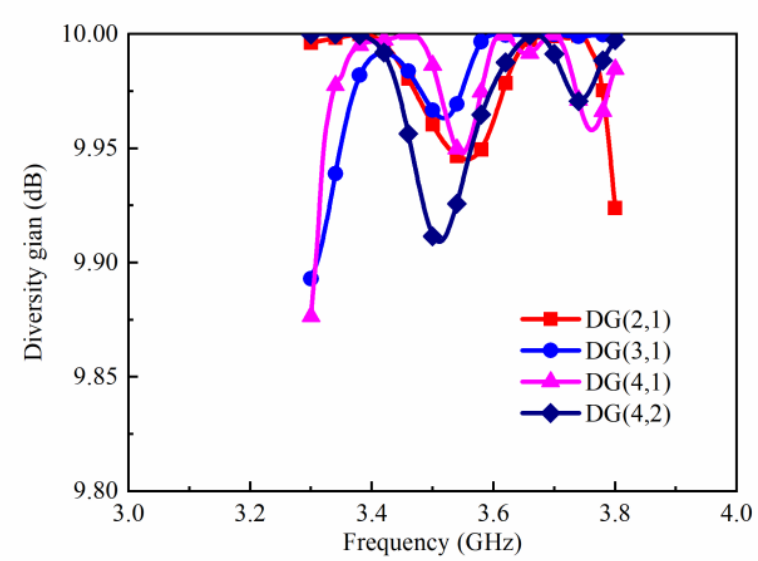

Fig. 24. Calculated DGs for the fabricated $8 \times 8$ MIMO antennas.

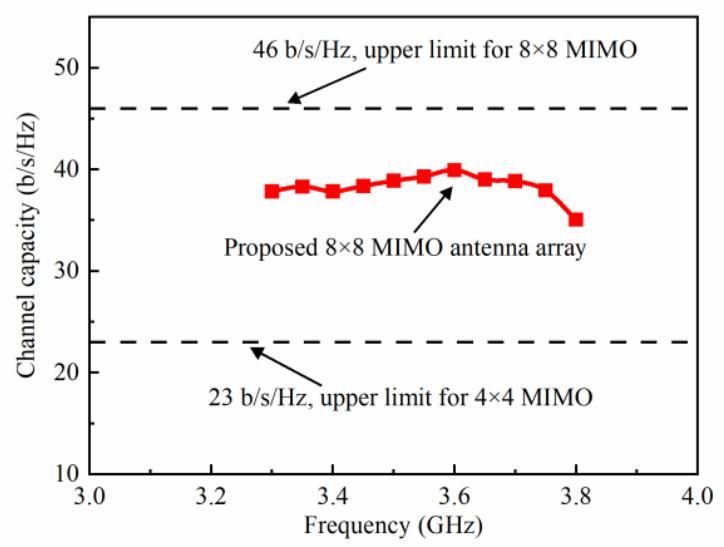

Fig. 25. Calculated ergodic channel capacities for the fabricated $8 \times 8$ MIMO antennas.

The simulated MEGs of the proposed MIMO antenna system is shown in Fig. 19. The MEGs of the antenna elements are nearly identical over the whole operating band, which suggests a good channel performance.

To have good quality and reliability in wireless systems, the diversity gain (DG) of the MIMO design should be close to 10 $\mathrm{dB}$ in the operating bandwidth. The DGs of the proposed MIMO antennas are simulated in Fig. 20. It can be observed that the DGs of the antennas is very stable with a range of 9.86 $\mathrm{dB}-10 \mathrm{~dB}$ in the entire operating range.

\section{B. Experimental Verification of $8 \times 8$ MIMO Antenna}

The proposed $8 \times 8$ MIMO antenna array was fabricated and measured. The photos of the fabricated antenna array are shown in Fig. 21. Fig. 22 shows the measured S-parameters of the fabricated prototype. It shows that the impedance bandwidth of the MIMO antenna array can cover 3.3-3.8 GHz. The measured transmission coefficients of $\left|S_{31}\right|,\left|S_{41}\right|$ and $\left|S_{32}\right|$ are lower than $-24 \mathrm{~dB}$. The isolation of the array is mainly limited by the isolation between the adjacent port 1 and port 2, which is still better than $20 \mathrm{~dB}$. The measured radiation efficiency and ECC for the fabricated $8 \times 8$ MIMO antenna array are shown in Fig. 23 . The measured results agree well with the simulated results. The antenna efficiency of the fabricated $8 \times 8$ MIMO antenna array is higher than $40 \%$ and the ECCs at different ports are 

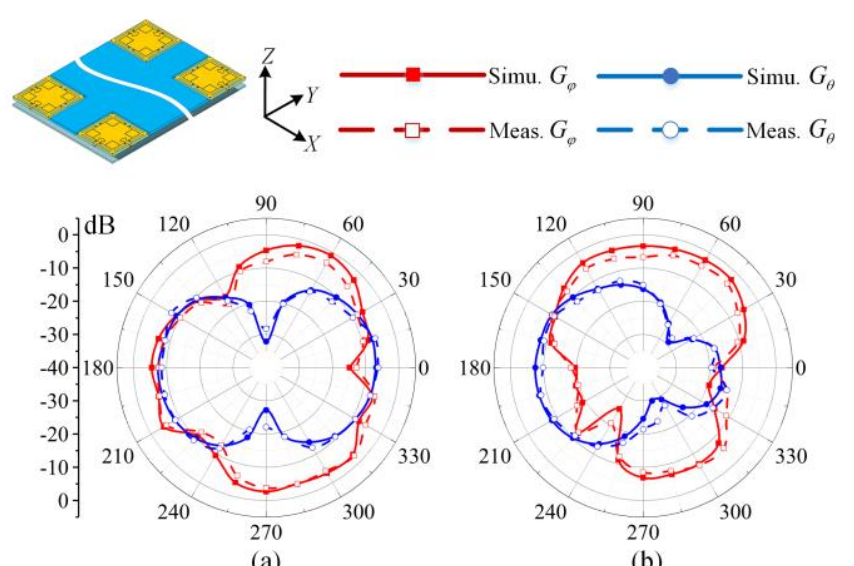

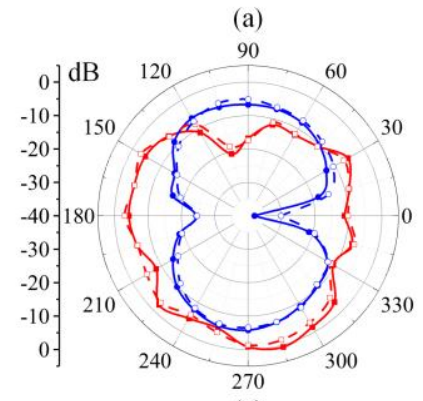

(c)

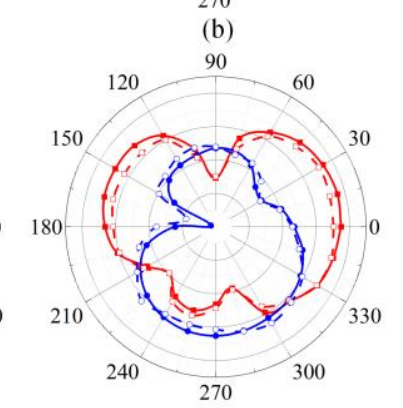

(d)
Fig. 26. Simulated and measured radiation patterns in the $x y$-plane of the fabricated antenna array. (a) Port 1 at 3.4GHz. (b) Port 1 at 3.8GHz. (c) Port 2 at $3.4 \mathrm{GHz}$. (d) Port 2 at $3.8 \mathrm{GHz}$.

lower than 0.13. Fig. 24 shows calculated DGs for the fabricated $8 \times 8$ MIMO antennas, which has an excellent diversity gain close to $10 \mathrm{~dB}$. Besides, the calculated ergodic channel capacities of the fabricated eight-element array are shown in Fig. 25. The channel capacities with a 20-dB SNR vary from 35 to $40 \mathrm{~b} / \mathrm{s} / \mathrm{Hz}$ in the operating bands, which are 1.5 times larger than the upper limit of a $4 \times 4$ MIMO system. Compared with the upper limit of $46 \mathrm{~b} / \mathrm{s} / \mathrm{Hz}$ for an $8 \times 8 \mathrm{MIMO}$ antennas, the proposed array exhibits desirable channel capacities that are only $11 \mathrm{~b} / \mathrm{s} / \mathrm{Hz}$ less than the ideal case. Therefore, the proposed wideband $8 \times 8$ MIMO array is capable of providing good MIMO performances for $5 \mathrm{G}$ terminal applications.

The simulated and measured radiation patterns at $3.4 \mathrm{GHz}$ and $3.8 \mathrm{GHz}$ are shown in Fig. 26. Due to the symmetrical array configuration, only radiation patterns excited by port 1 and port 2 are given for brevity. As can be observed, the measured radiation patterns agree well with the simulated results. The radiation patterns of the MIMO antenna array are approximately omnidirectional, which is highly desirable for terminal equipment. The radiation performance measurement was carried out in a near-field antenna measurement system at Xidian University.

\section{User's Hand Effects}

To investigate the operating performances of the MIMO antenna array in real practical scenarios, the effects of the user's hand are studied in the subsection. As shown in Fig. 27(a) and Fig. 27(b), two typical 5G usage scenarios are investigated, including single-hand operation (SHO) and dual-hand operation (DHO).
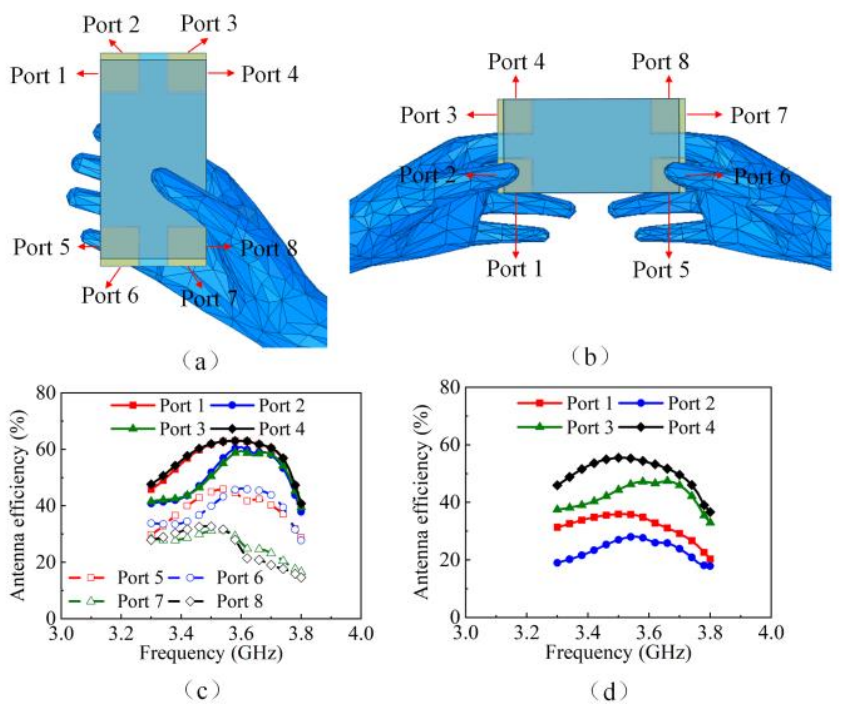

Fig. 27. Two typical usage scenarios under user's operation and simulated antenna efficiencies of the proposed $8 \times 8$ MIMO antennas. (a) SHO mode. (b) DHO mode. (c) Antenna efficiencies in SHO mode. (d) Antenna efficiencies in DHO mode.

Normally, S-parameters are less affected by the user's hand [10]. However, the antenna efficiency of the proposed array will be affected significantly owing to the absorption effect of the user's hand. For SHO mode shown in Fig. 27(c), the antenna efficiencies of port 7 and port 8 are declined to $15 \%$ when the antenna is close to the hand. In addition, the antenna efficiencies of port 5 and port 6 are reduced slightly. For DHO mode, a similar phenomenon can be observed in Fig. 27(d). The antenna efficiencies of only $18 \%$ are obtained. Therefore, the closer the antenna elements are to the user's hand, the greater effects on the radiation efficiency of the antenna will be expected.

\section{Comparison}

Table I gives the comparison between the proposed MIMO antennas and the conventional antenna designs in [19] [24]. Although the MIMO antenna pairs with a shared radiator in [19] can realize broad bandwidth, the isolation is around $12 \mathrm{~dB}$. The MIMO performance of the antennas will be affected by their low isolation. In addition, the 7.5-mm thickness and $3.2-\mathrm{mm}$ floor headroom are difficult to use in actual applications where smartphone devices are tightly packed. The shared-radiator MIMO antenna pairs have a wide impedance band, however, 10-dB isolation makes it difficult to reduce crosstalk of signals between ports [24].

The broadband MIMO antennas design is achieved based on multiple CMs, but the isolation is only $12.5 \mathrm{~dB}$ [28]. Although wideband MIMO antennas with tri-band operation are achieved based on multiple CMs [29], CMSRRs are used to improve the isolation between MIMO antennas, which occupy a large space inside the smartphone with a thickness of $3.2 \mathrm{~mm}$ and a ground clearance of $4 \mathrm{~mm}$. The dual-band high-isolation MIMO antennas are proposed based on orthogonal two sets of CMs [33], but it has a narrow bandwidth and does not have the shared radiator to improve space utilization. A high-isolation MIMO antenna pair based on a pair of orthogonal CMs has a 
TABLE I

COMPARISON OF THE DECOUPLED MIMO ANTENNAS FOR 5G TERMINAL APPLICATIONS

\begin{tabular}{|c|c|c|c|c|c|c|c|}
\hline Ref. & $\begin{array}{c}\text { Shared } \\
\text { Radiator }\end{array}$ & $\begin{array}{c}\text { Impedance Bandwidth } \\
(<-6 \mathrm{~dB})\end{array}$ & Isolation & $\begin{array}{c}\text { Isolation } \\
\text { Technique } \\
\end{array}$ & Efficiency & Thickness & $\begin{array}{c}\text { Ground } \\
\text { Clearance } \\
\end{array}$ \\
\hline$[19]$ & $\sqrt{ }$ & $3.3-5.0 \mathrm{GHz}(40.9 \%)$ & $>12 \mathrm{~dB}$ & Orthogonal modes & $>31.6 \%$ & $\begin{array}{c}7.5 \mathrm{~mm} \\
\left(0.082 \lambda_{0}\right)\end{array}$ & $\begin{array}{c}3.2 \mathrm{~mm} \\
\left(0.035 \lambda_{0}\right)\end{array}$ \\
\hline$[28]$ & $x$ & $\begin{array}{l}3.31-3.70 \mathrm{GHz}(11.1 \%) \\
4.46-5.40 \mathrm{GHz}(19.1 \%)\end{array}$ & $>12.5 \mathrm{~dB}$ & l & $\begin{array}{l}>42.5 \% \\
>81.1 \% \\
\end{array}$ & $\begin{array}{c}0.8 \mathrm{~mm} \\
\left(0.009 \lambda_{0}\right)\end{array}$ & $\begin{array}{c}3 \mathrm{~mm} \\
\left(0.033 \lambda_{0}\right)\end{array}$ \\
\hline$[33]$ & $x$ & $\begin{array}{l}27.6-28.3 \mathrm{GHz}(2.5 \%) \\
37.2-39.0 \mathrm{GHz}(4.7 \%)\end{array}$ & $>28 \mathrm{~dB}$ & $\begin{array}{l}\text { Orthogonal two sets of CMs } \\
\text { with dual band }\end{array}$ & $>88.8 \%$ & / & l \\
\hline$[34]$ & $\sqrt{ }$ & $2.4-2.49 \mathrm{GHz}(3.7 \%)$ & $>20 \mathrm{~dB}$ & $\begin{array}{c}\text { Orthogonal a pair of CMs with } \\
\text { narrow band }\end{array}$ & $>75 \%$ & $\begin{array}{c}7 \mathrm{~mm} \\
\left(0.056 \lambda_{0}\right) \\
\end{array}$ & $\begin{array}{c}3 \mathrm{~mm} \\
\left(0.024 \lambda_{0}\right)\end{array}$ \\
\hline$[35]$ & $x$ & $4.2-8.0 \mathrm{GHz}(65.5 \%)$ & $>25 \mathrm{~dB}$ & Metamaterial unit cells & $>70 \%$ & $\begin{array}{c}1.6 \mathrm{~mm} \\
\left(0.022 \lambda_{0}\right)\end{array}$ & $\begin{array}{l}13.25 \mathrm{~mm} \\
\left(0.186 \lambda_{0}\right)\end{array}$ \\
\hline
\end{tabular}

* CM: Characteristic mode.

* CMSRR: Complimentary modified split ring resonator.

$* \lambda_{0}$ : The wavelength at the lowest operating frequency in free space.

narrow band in [34], but it occupies a large profile of 7-mm thickness and 3-mm ground clearance. Ref. [35] presents a wideband high-isolation two-element MIMO antenna using metamaterial unit cells. But it has a large ground clearance, and is difficult to be integrated into smartphones. A broadside tri-modal MIMO patch antenna based on bandwidth enhancement technology is developed in [36]. However, its 9-mm antenna profile is thicker than the thickness of typical smartphones. These two CMA-based MIMO antennas do not have a good trade-off between high performance and miniaturization for terminal applications. The proposed back-cover dual-antennas pair based on two sets of orthogonal CMs has an overall high performance with compactness (shared radiator), broadband (two sets), high isolation (orthogonal $\mathrm{CMs}$ ), low profile $(1.6 \mathrm{~mm})$ and zero ground clearance, which fully meets the practical needs for terminal applications.

\section{CONCLUSION}

In this paper, a novel method to realize a designing a wideband high-isolation MIMO antenna pair with a shared radiator based on dual sets of CMs is presented. Due to the orthogonality of the two sets of CMs, the isolation of the MIMO dual-antenna pair can be enhanced to $20 \mathrm{~dB}$ without using any additional decoupling structures. In addition, a wide impedance bandwidth covering 3.3-3.8 GHz is obtained. The antenna pairs are integrated with a dielectric back cover and a system circuit board to form an $8 \times 8$ MIMO antenna array. Detailed analyses and results show that $20-\mathrm{dB}$ isolation, radiation efficiencies greater than $40 \%$, and the ECCs less than 0.13 are achieved for the designed MIMO antenna array, which meets the practical needs of $5 \mathrm{G}$ terminal antennas. Good agreement was obtained between the simulated and the measured results. Compared to other published MIMO antennas, the presented antenna has a compact size and leaves more design space for other antennas and components. The proposed antenna is a promising candidate for terminal applications in 5G and future mobile devices.

\section{REFERENCES}

[1] J. G. Andrews et al., "What will 5G be?" IEEE J. Sel. Areas Commun., vol. 32, no. 6, pp. 1065-1082, Jun. 2014.

[2] A. Kumar, K. Mishra, A. Mukherjee, and A. K. Chaudhary, "Channel capacity enhancement using MIMO technology," IEEE-International Conference On Advances In Engineering, Science And Management (ICAESM -2012), Nagapattinam, Tamil Nadu, 2012, pp. 10-15.

[3] Z. Zhang, Antenna Design for Mobile Devices, 2nd ed. Hoboken, NJ, USA: Wiley, 2017.

[4] Y. Wang and Z. Du, "A printed dual-antenna system operating in the GSM1800/ GSM1900/ UMTS/ LTE2300/ LTE2500/ 2.4-GHz WLAN bands for mobile terminals," IEEE Antennas Wireless Propag. Lett., vol. 13, pp. 233-236, 2014.

[5] Y.-L. Ban, Z.-X. Chen, Z. Chen, K. Kang, and J. L.-W. Li, "Decoupled closely spaced heptaband antenna array for WWAN/LTE smartphone applications," IEEE Antennas Wireless Propag. Lett., vol. 13, pp. 31-34, 2014.

[6] J.-Y. Lu, H.-J. Chang, and K.-L. Wong, "10-antenna array in the smartphone for the 3.6-GHz MIMO operation," in Proc. IEEE Int. Symp. Antennas Propag. USNC/URSI Nat. Radio Sci. Meeting, Vancouver, BC, Canada, Jul. 2015, pp. 1220-1221.

[7] Y. Li, C.-Y.-D. Sim, Y. Luo, and G. Yang, "12-port 5G massive MIMO antenna array in sub-6GHz mobile handset for LTE bands $42 / 43 / 46$ applications," IEEE Access, vol. 6, pp. 344-354, 2018.

[8] W. Hu, X. Liu, S. Gao, L.-H. Wen, L. Qian, T. Feng, R. Xu, P. Fei, and Y. Liu, "Dual-band ten-element MIMO array based on dual-mode IFAs for 5G terminal applications," IEEE Access, vol. 7, pp. 178476-178485, 2019.

[9] J. Guo, L. Cui, C. Li, and B. Sun, "Side-edge frame printed eight-port dual-band antenna array for 5G smartphone applications," IEEE Trans. Antennas Propag., vol. 66, no. 12, pp. 7412-7417, Dec. 2018.

[10] W. Hu, L. Qian, S. Gao, L.-H. Wen, Q. Luo, H. Xu, X. Liu, Y. Liu, and W. Wang, "Dual-band eight-element MIMO array using multi-slot decoupling technique for 5G terminals," IEEE Access, vol. 7, pp. 153910-153920, 2019. 
[11] L. Cui, J. Guo, Y. Liu and C.-Y.-D. Sim, “An 8-element dual-band MIMO antenna with decoupling stub for 5G smartphone applications," IEEE Antennas Wireless Propag. Lett., vol. 18, pp. 2095-2099, Oct. 2019.

[12] A. Zhao and Z. Ren, "Size reduction of self-isolated MIMO antenna system for 5G mobile phone applications," IEEE Antennas Wireless Propag. Lett., vol. 18, pp. 152-156, Jan. 2019.

[13] Y. Li, C. Y. D. Sim, Y. Luo, and G. Yang, "High-isolation $3.5 \mathrm{GHz}$ eight -antenna MIMO array using balanced open-slot antenna element for $5 \mathrm{G}$ smartphones," IEEE Trans. Antennas Propag., vol. 67, no. 6, pp. 3820-3830, Jun. 2019.

[14] M.-Y. Li et al., "Eight-port orthogonally dual-polarized antenna array for 5G smartphone applications," IEEE Trans. Antennas Propag., vol. 64, no. 9, pp. 3820-3830, Sep. 2016.

[15] X. Zhao, S. P. Yeo, and L. C. Ong, "Decoupling of inverted-F antennas with high-order modes of ground plane for 5G mobile MIMO platform," IEEE Trans. Antennas Propag., vol. 66, no. 9, pp. 4485-4495, Sep. 2018.

[16] L. Chang, Y. Yu, K. Wei, and H. Wang, "Orthogonally polarized dual antenna pair with high isolation and balanced high performance for $5 \mathrm{G}$ MIMO smartphone," IEEE Trans. Antennas Propag., vol. 68, no. 5, pp. 3487 -3495, May 2020.

[17] M.-Y. Li, Z.-Q. Xu, Y.-L. Ban, C.-Y.-D. Sim, and Z.-F. Yu, "Eight-port orthogonally dual-polarized MIMO antennas using loop structures for $5 \mathrm{G}$ smartphone," IET Microw. Antennas Propag., vol. 11, pp. 1810-1816, Sep. 2017.

[18] L. Chang, Y. Yu, K. Wei, and H. Wang, "Polarization-orthogonal co-frequency dual antenna pair suitable for 5G MIMO smartphone with metallic bezels," IEEE Trans. Antennas Propag., vol. 67, no. 8, pp. 5212-5220, Aug. 2019.

[19] L. Sun, Y. Li, Z. Zhang, and Z. Feng, "Wideband 5G MIMO antenna with integrated orthogonal-mode dual-antenna pairs for metal-rimmed smartphones," IEEE Trans. Antennas Propag., vol. 68, no. 4, pp. 2494-2503, Apr. 2020.

[20] H. Xu, S. Gao, H. Zhou, H. Wang, and Y. Cheng, "A highly integrated MIMO antenna unit: Differential/common mode design," IEEE Trans. Antennas Propag., vol. 67, no. 11, pp. 6724-6734, Nov. 2019.

[21] L. Sun, Y. Li, Z. Zhang, and H. Wang, "Self-decoupled MIMO antenna pair with shared radiator for 5G smartphones," IEEE Trans. Antennas Propag., vol. 68, no. 5, pp. 3423-3432, May 2020.

[22] Z. Ren, A. Zhao, and S. Wu, "MIMO antenna with compact decoupled antenna pairs for 5G mobile terminals," IEEE Antennas Wireless Propag. Lett., vo 1. 18, pp. 1367-1371, Jul. 2019.

[23] C. Deng, D. Liu, and X. Lv, "Tightly arranged four-element MIMO antennas for $5 \mathrm{G}$ mobile terminals," IEEE Trans. Antennas Propag., vol. 67, no. 10, pp. 6353-6361, Oct. 2019.

[24] I. R. R. Barani, K.-L. Wong, Y.-X. Zhang, and W.-L. Li, "Low-profile wideband conjoined open-slot antennas fed by grounded coplanar waveguides for $4 \times 4$ 5G MIMO operation," IEEE Trans. Antennas Propag., vol. 68, no. 4, pp. 2646-2657, Apr. 2020.

[25] R. J. Garbacz and R. Turpin, "A generalized expansion for radiated and scattered fields," IEEE Trans. Antennas Propag., vol. 19, no. 3, pp. 348358, May 1971.

[26] R. F. Harrington and J. R. Mautz, "Theory of characteristic modes for conducting bodies," IEEE Trans. Antennas Propag., vol. AP-19, no. 5, pp. 622-628, Sep. 1971.

[27] X. Zhao, S. P. Yeo, and L. C. Ong, "Planar UWB MIMO antenna with pattern diversity and isolation improvement for mobile platform based on the theory of characteristic modes," IEEE Trans. Antennas Propag., vol. 66, no. 1, pp. 420-425, Jan. 2018.

[28] J. Dong, S. Wang, and J. Mo, "Design of a twelve-port MIMO antenna system for multi-mode 4G/5G smartphone applications based on characteristic mode analysis," IEEE Access, vol. 8, pp. 90751-90759, 2020 .

[29] N. Kumar and R. Khanna. "A compact multi-band multi-input multi -output antenna for $4 \mathrm{G} / 5 \mathrm{G}$ and IoT devices using theory of characteristic modes," Int J RF Microw Comput Aided Eng, 30: e22012, 2020.

[30] R. Xu, S. Gao, J. Liu, J. Y. Li, Q. Luo, W. Hu, L. H. Wen, X. X. Yang, and J. T. S. Sumantyo, "Analysis and design of ultrawideband circularly polarized antenna and array," IEEE Trans. Antennas Propag., vol. 68, no. 12, pp. 7842-7853, Dec. 2020.

[31] M. Bouezzeddine and W. L. Schroeder, "Design of a wideband, tunable four-port MIMO antenna system with high isolation based on the theory of characteristic modes," IEEE Trans. Antennas Propag., vol. 64, no. 7, pp. 2679-2688, Jul. 2016.
[32] K. S. Sultan, H. H. Abdullah, E. A. Abdallah, and H. S. El-Hennawy, "Metasurface-based dual polarized MIMO antenna for 5G smartphones using CMA," IEEE Access, vol. 8, pp. 37250-37264, 2020.

[33] K. S. Jayasree, P.V.Y., and V. Saradhi. "Orthogonal mode dual band MIMO antenna system for 5G smartphone applications using characteristic mode analysis," Circuit World, Vol. 47 No. 3, pp. 284-289, 2021.

[34] D. Wen, Y. Hao, H. Wang, and H. Zhou, "Design of a MIMO antenna with high isolation for smartwatch applications using the theory of characteristic modes," IEEE Trans. Antennas Propag., vol. 67, no. 3, pp. 1437-1447, Mar. 2019.

[35] A. H. Jabire, H. X. Zheng, A. Abdu, and Z. Song. "Characteristic mode analysis and design of wide band MIMO antenna consisting of metamaterial unit cell." Electronics, vol. 8, no. 1, p. 68, 2019.

[36] C. Y. Chiu, B. K. Lau, and R. Murch, "Bandwidth enhancement technique for broadside tri-modal patch antenna," IEEE Open J. Antennas Propag., vol. 1, pp. 524-533, 2020.

[37] R. Martens, E. Safin, and D. Manteuffel, "Inductive and capacitive excitation of the characteristic modes of small terminals," Proc. Loughborough Antennas Propag. Conf., Loughborough, U.K., Nov. 2011, pp. $1-4$.

[38] F. W. Yao and S. S. Zhong, "Broadband slot antenna with a fan-shaped microstrip feed," 2005 IEEE International Symposium on Microwave, Antenna, Propagation and EMC Technologies for Wireless Communications, Beijing, China, 2005, pp. 127-130 Vol. 1.

[39] Y. Liu, W. Zhou, S. Yang, W. Li, P. Li, and S. Yang, "A novel miniaturized vivaldi antenna using tapered slot edge with resonant cavity structure for ultrawideband applications," IEEE Antennas Wireless Propag. Lett., vol. 15, pp. 1881-1884, 2016.

[40] Y. Liu, A. Ren, H. Liu, H. Wang, and C.-Y. Sim, "Eight-port MIMO array using characteristic mode theory for $5 \mathrm{G}$ smartphone applications," IEEE Access, vol. 7, pp. 45679-45692, 2019.

[41] J. Zhu, S. Li, B. Feng, L. Deng, and S. Yin, "Compact dual-polarized UWB quasi-self-complementary MIMO/diversity antenna with bandrejection capability," IEEE Antennas Wireless Propag. Lett., vol. 15, pp. 905-908, 2016.

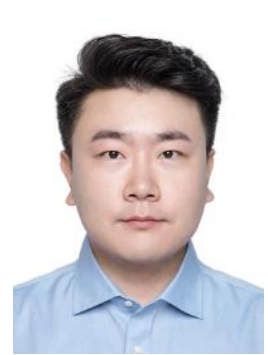

Wei Hu (Member, IEEE) received the $\mathrm{Ph} . \mathrm{D}$. degree in electromagnetic fields and microwave technology from Xidian University, Xi'an, China, in 2013. From 2013 to 2017, he was a lecturer with the National Key Laboratory of Antennas and Microwave Technology, Collaborative Innovation Center of Information Sensing and Understanding, Xidian University, where he is currently an Associate Professor. From 2018 to 2019, he visited the University of Kent, Kent, U.K., as an Academic Visitor. He has authored or coauthored over 80 internationally refereed journal articles and has been serving as a reviewer for a number of technical journals and international conferences. His current research interests include multiband and wideband antennas, circularly polarized antennas, MIMO antenna arrays, and wideband wide-scanning phased arrays.

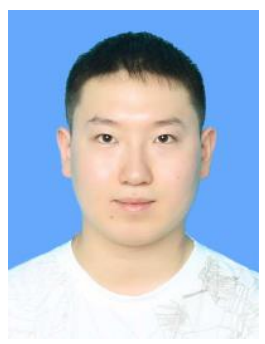

Zhan Chen was born in Chongqing, China, in September 1996. He received the B.S. degree from Xidian University, Xi' an, China, in 2019. He is currently pursuing the Ph.D. degree in electromagnetic field and microwave technology from the National Key Laboratory of Antennas and Microwave Technology, Xidian University, Xi'an, China. His research interests include 5G MIMO antennas, wideband circularly polarized antennas and wideband wide-scanning phased arrays. 


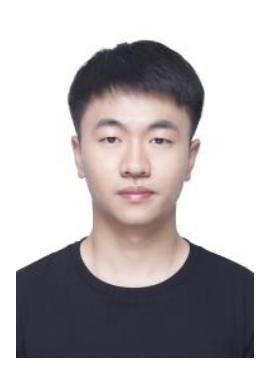

Long Qian received the B.S. and M.S. degree from Xidian University, Xi'an, China, in 2017 and 2020. He is currently pursuing the Ph.D. degree in the School of Electronic Engineering and Computer Science, Queen Mary University of London. His research interests include mobile terminal antennas and 5G MIMO antennas for wireless applications.

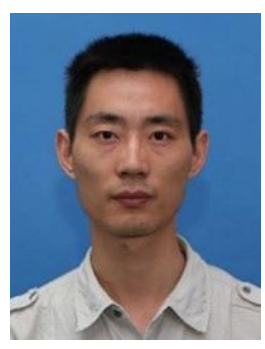

Lehu Wen (Member, IEEE) received the Ph.D. degree in electronic engineering from the University of Kent, Canterbury, U.K., in 2020. He is currently a Research Associate with the School of Engineering and Digital Arts, University of Kent. His research interests include wideband dual-polarized antennas, circularly polarized antennas, mobile terminal antennas, and tightly coupled array antennas.

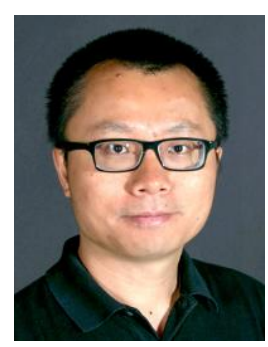

Qi Luo (Senior Member, IEEE) received the M.Sc. degree (Hons.) from the University of Sheffield, Sheffield, U.K., in 2006, and the Ph.D. degree (Hons.) from the University of Porto, Porto, Portugal, in 2012. From 2012 to 2013, he was with the Surrey Space Center, Guildford, U.K., as a Research Fellow. From 2013 to 2020, he was with the School of Engineering and Digital Arts, University of Kent, Canterbury, U.K., as a Research Fellow. He is currently a Senior Lecturer with the Department of Engineering and Technology, University of Hertfordshire, Hatfield, U.K. He has authored or coauthored two books Circularly Polarized Antennas (Wiley-IEEE, 2014) and Low-Cost Smart Antennas (Wiley, 2019). He also authored a book chapter in Handbook of Antenna Technologies (Singapore: Springer, 2014). His current research interests include smart antennas, circularly polarized antennas, reflectarray, transmitarray, multiband microstrip antennas, and electrically small antenna design.

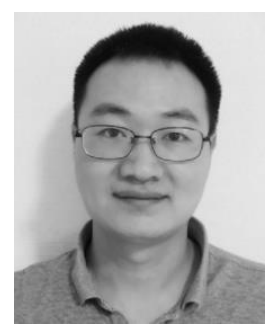

Rui Xu (Member, IEEE) was born in Xi'an, China, in 1989. He received the B.E., master's, and Ph.D. degrees in electronic engineering from Northwestern Polytechnical University, Xi'an, in 2013, 2015, and 2018, respectively. From 2019 to 2020, he was a Research Associate with the University of Kent, Kent, U.K. Now, he is a Research Fellow with Nanyang Technological University (NTU), Singapore. His current research is in ultrawideband antennas, waveguide slot antennas, $\mathrm{THz}$ antennas, print slot antennas, microstrip antennas, high-gain antennas, and electromagnetic periodic structure. Dr. $\mathrm{Xu}$ has been serving as a reviewer for several technical journals and international conferences.

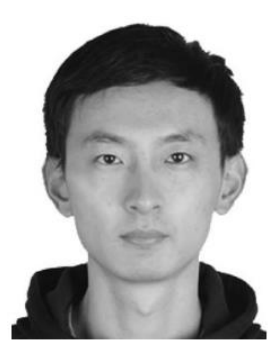

Wen Jiang (Member, IEEE) was born in Shandong, China, in November 1985. He received the B.S. and Ph.D. degrees from Xidian University, Xi'an, China, in 2008 and 2012, respectively. He is currently the Vice Director with the National Key Laboratory of Science and Technology on Antennas and Microwaves, Xidian University. He is also a Full Professor with Xidian University. His current research interests include electromagnetic scattering theory and technology, antenna theory and engineering, and electromagnetic measurement theory and technology.

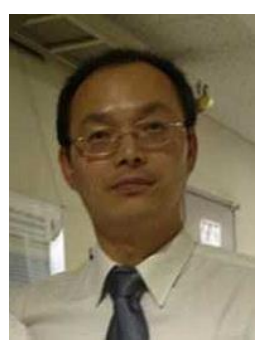

Steven Gao (Fellow, IEEE) received the $\mathrm{Ph} . \mathrm{D}$. degree in Microwave Engineering from Shanghai University, P.R. China. He is a Full Professor and Chair in RF and Microwave Engineering, and the Director of Graduate Studies at the School of Engineering and Digital Arts, University of Kent, UK. His research covers smart antennas, phased arrays, MIMO, reconfigurable antennas, wideband/multiband antennas, satellite antennas, $\mathrm{RF} / \mathrm{microwave} / \mathrm{mm}$-wave $/ \mathrm{THz}$ circuits, mobile communications, satellite communications, UWB radars, synthetic-aperture radars, IOT and sensors for healthcare. He co-authored/co-edited three books (Space Antenna Handbook, Wiley, 2012; Circularly Polarized Antennas, IEEE-Wiley, 2014; Low-Cost Smart Antennas, Wiley, 2019), over 300 papers and 10 patents. He was a Distinguished Lecturer of IEEE AP Society, and is currently an Associate Editor of IEEE Transactions on Antennas and Propagation and several other international Journals (Radio Science, IEEE Access, Electronics Letters, IET Circuits, Devices and Systems, etc.), and the Editor-in-Chief for John Wiley \& Sons Book Series on "Microwave and Wireless Technologies". He was General Chair of LAPC 2013, and an Invited Speaker at many conferences. He is a Fellow of IEEE, and also a Fellow of Royal Aeronautical Society, UK, and the IET, UK. 